\title{
Gastrointestinal and Genitourinary Toxicity Profiles of Metformin Versus Placebo in Men With Prostate Cancer Receiving Pelvic Radiotherapy: Interim Toxicity Results of a Double-blinded, Multicenter, Phase II Randomized Controlled Trial
}

\section{Nawaid Usmani}

University of Alberta

\section{Megan O. McDonald}

University of Manitoba Faculty of Medicine: University of Manitoba Max Rady College of Medicine

\section{Aldrich Ong}

University of Manitoba Faculty of Medicine: University of Manitoba Max Rady College of Medicine

\section{Rashmi Koul}

University of Manitoba Faculty of Medicine: University of Manitoba Max Rady College of Medicine Arbind Dubey

University of Manitoba Faculty of Medicine: University of Manitoba Max Rady College of Medicine William Hunter

University of Manitoba Faculty of Medicine: University of Manitoba Max Rady College of Medicine Shahida Ahmed

University of Manitoba Faculty of Medicine: University of Manitoba Max Rady College of Medicine Harvey Quon

University of Calgary Faculty of Medicine: University of Calgary Cumming School of Medicine

\section{Don Yee}

University of Alberta

\section{Matthew Parliament}

University of Alberta

\section{Gokulan Sivananthan}

University of Manitoba Faculty of Medicine: University of Manitoba Max Rady College of Medicine

\section{Brita Danielson}

University of Alberta

\section{Lindsay Rowe}

University of Alberta

\section{Sunita Ghosh}

University of Alberta 


\section{Julian 0. Kim ( $\nabla_{\text {jkim7@@cancercare.mb.ca ) }}$}

CancerCare Manitoba https://orcid.org/0000-0003-0332-2024

\section{Short report}

Keywords: Metformin, Prostate Cancer, Gastrointestinal Toxicity, Genitourinary Toxicity

Posted Date: February 23rd, 2021

DOI: https://doi.org/10.21203/rs.3.rs-237354/v1

License: (c) (1) This work is licensed under a Creative Commons Attribution 4.0 International License. Read Full License 


\section{Abstract}

Androgen deprivation therapy (ADT) used for prostate cancer (PCa) management is associated with metabolic and anthropometric toxicity. Metformin given concurrent to ADT is hypothesized to counteract these changes. This planned interim analysis reports the gastrointestinal and genitourinary toxicity profiles of PCa patients receiving ADT and prostate/pelvic radiotherapy plus metformin versus placebo as part of a phase 2 randomized controlled trial. Men with intermediate or high-risk PCa were randomized 1:1 to metformin versus placebo. Both groups were given ADT for 18-36 months with minimum 2-month neoadjuvant phase prior to radiotherapy. Acute gastrointestinal and genitourinary toxicities were quantified using CTCAE v4.0. Differences in $\geq$ grade 2 toxicities by treatment were assessed by chisquared test. 83 patients were enrolled with 44 patients randomized to placebo and 39 randomized to metformin. There were no significant differences at any time point in $\geq$ grade 2 gastrointestinal toxicities or overall gastrointestinal toxicity. Overall $\geq$ grade 2 gastrointestinal toxicity was low prior to radiotherapy (7.9\% (placebo) vs. $3.1 \%$ (metformin), $p=0.39$ ) and at the end of radiotherapy ( $2.8 \%$ (placebo) vs $3.1 \%$ (metformin), $p=0.64)$. There were no differences in overall $\geq$ grade 2 genitourinary toxicity between treatment arms (19.0\% (placebo) vs. 9.4\% (metformin), $p=0.30$ ). Metformin added to radiotherapy and ADT did not increase rates of $\geq$ grade 2 gastrointestinal or genitourinary toxicity and is generally safe and well-tolerated.

\section{Background}

Androgen deprivation therapy (ADT) , a cornerstone of modern prostate cancer (PCa) management ${ }^{1}$, is associated with improved survival amongst men with high-risk prostate cancer (PCa) when added to radiotherapy ${ }^{2-5}$. However, ADT induced hypogonadism is associated with metabolic derangements (dyslipidemia ${ }^{6}$, hyperinsulinemia ${ }^{7,8}$, and insulin resistance ${ }^{6-8}$ ) and anthropometric changes (weight gain, centralized obesity ${ }^{9,10}$ ) which can lead to metabolic syndrome, diabetes, and cardiovascular disease. ${ }^{11}$

Metformin, an economical anti-hyperglycemic medication, is known to decrease/stabilize weight, decrease plasma triglycerides, and reduce diabetes incidence and complications. ${ }^{12,13}$ There is considerable interest in employing adjunctive metformin to potentially counteract the metabolic and anthropometric changes associated with ADT, and potentially improve PCa outcomes. ${ }^{14,15}$

Metformin is associated with mild/moderate gastrointestinal ( $\mathrm{Gl}$ ) side effects that ameliorate with dose titration. ${ }^{12,13}$ Approximately $20 \%$ of patients will experience diarrhea, abdominal discomfort, anorexia, nausea, or bloating during metformin initiation. ${ }^{12,13}$ The toxicity of metformin concurrent to prostate/pelvic radiotherapy and ADT is unknown. This planned interim analysis of the PREMIUM trial (Prevention of Metabolic Syndrome and Increased Weight Using Metformin Concurrent to Androgen Deprivation Therapy for Locally Advanced Adenocarcinoma of the prostate, Clinicaltrials.gov trial identifier NCT01996696), reports the gastrointestinal and genitourinary toxicity profiles of high-risk PCa 
patients undergoing ADT and prostate/pelvic radiotherapy plus metformin versus placebo on a phase 2 randomized controlled trial (RCT).

\section{Methods}

Patient Selection: Eligible patients were males $\geq 18$ years old, Eastern Cooperative Oncology Group (ECOG) 0 to 1 and normoglycemic (Fasting Plasma Glucose $\leq 6.9 \mathrm{mmol} / \mathrm{L}$ or HemoglobinA1C (HgbA1C) $<6.5 \%$ ) with biopsy confirmed High Tier Intermediate risk ( $\geq 2$ of: Gleason Score (GS) = 7, PSA of 10-20 $\mathrm{ng} / \mathrm{mL}$, or $\geq 50 \%$ of biopsy cores containing GS 7) or high-risk PCa (any of: T3 disease, GS $\geq 8.0$, and/or $P S A \geq 20 \mathrm{ng} / \mathrm{mL}$ ) receiving curative intent ADT and external beam radiotherapy (EBRT).

Study Design \& Treatments: Participants were randomized (1:1), stratified by treatment center, to metformin $500 \mathrm{mg}$ by mouth (PO) 3 times daily for 30 to 36 months or identical placebo. Metformin was titrated as follows: $500 \mathrm{mg}$ by PO daily for two weeks, then $500 \mathrm{mg}$ PO twice daily for two weeks, then $500 \mathrm{mg}$ PO three times daily for the remainder of treatment. Study drugs were initiated 2 months (minimum) prior to the start of radiation. Both groups were given luteinizing hormone-releasing hormone (LHRH) agonist injections for 18-36 months with a minimum 2-month neoadjuvant phase prior to EBRT. RT consisting of $46 \mathrm{~Gy} / 23$ \# or $50.4 \mathrm{~Gy} / 28$ \# was delivered to pelvic lymph nodes (recommended by protocol) plus prostate boost to a total of $78 \mathrm{~Gy} / 39$ \# (or interstitial brachytherapy boost to $110-115 \mathrm{~Gy}$ or hypofractionated EBRT equivalent). Acceptable EBRT total prostate doses included: 70 Gy/28\#, 68 Gy/25\#, or $60 \mathrm{~Gy} / 20 \#$. All EBRT treatments utilized intensity modulated radiotherapy (IMRT) or volumetric modulated arc therapy (VMAT).

Statistical Considerations: Planned sample size was 104 patients ( $97 \%$ power, 2-tailed a of 0.05 to detect a $4 \mathrm{~kg}$ difference in weight at 12 months). This pre-planned safety interim analysis was triggered after 52 patients completed 12 months follow-up to assess toxicity levels. Acute and subacute $\mathrm{GI}$ and GU toxicity was quantified using the Common Terminology Criteria for Adverse Events version 4.0 at: month 0 (baseline), month 3 (Pre-RT), month 5 (End of RT), and 12 months. Baseline characteristics were stratified by treatment arm, and differences in characteristics were assessed using standard parametric and nonparametric tests. Differences in $\geq$ grade 2 toxicities were assessed by chi-squared test.

\section{Results}

At the time of interim analysis (frozen for analysis 15/01/2020) 83 patients were enrolled between December 2015 and September 2019 at three participating centers with mean follow-up of 27.3 months (range 0.5-63.2). Fourty-four patients were randomized to placebo and 39 were randomized to metformin. Two patients randomized to receive placebo did not receive radiotherapy; one of which withdrew from study prior to radiotherapy and was lost to follow-up, and the other patient declined radiotherapy in favour of cryotherapy. Eighty-one patients were included for analysis (Figure 2). 
Baseline characteristics of the cohort included: mean age of 72 years (SD 7.1; range 49-86), mean body mass index $30.3 \mathrm{~kg} / \mathrm{m}^{2}$ (SD 5.5; range 22.2-52.5), median Gleason score 9 (range 7-9), and mean HgbA1C was 5.6\% (range 4.9-6.4) (Table 1). All patients completed RT, with most receiving EBRT prostate boosts total dose of 76-78Gy/38-39\# (62\% (Metformin arm), 57\% (placebo Arm)), or other hypofractionated schedules. Pelvic nodal radiation was used for most participants (71\% (Metformin arm), 79\% (placebo arm)). Interstitial brachytherapy boost was done in the minority (10\% (Metformin arm), $12 \%$ (placebo arm)). There were no statistically significant differences in baseline patient, disease, or treatment characteristics by arm.

Six patients randomized to receive metformin and 12 patients randomized to receive placebo discontinued the study drug prior to 12 months follow-up. Two patients discontinued early due to gastrointestinal side effects including: one patient from the placebo arm who discontinued at 4 months (during RT) due to grade 1 diarrhea, and one patient from the metformin arm who discontinued at 6 months (post-RT) due grade 1 bloating. All other patients who discontinued study drug did so at patient preference. No patients discontinued the study due to $\geq$ grade $3 \mathrm{GI}$ or GU toxicity.

Amongst the 81 participants analyzed, there were no significant difference in $\geq$ grade $2 \mathrm{Gl}$ toxicities including bloating, dyspepsia, nausea, diarrhea or overall GI toxicity at any time during follow-up (Figure 1 , Supplemental Table 1). Overall $\geq$ grade $2 \mathrm{GI}$ toxicity was low prior to RT ( $7.9 \%$ (placebo) vs. $3.1 \%$ Metformin), $p=0.39$ ) and at the end of RT ( $2.8 \%$ (placebo) vs 3.1\% (Metformin), $p=0.64$ ). Patients receiving metformin experienced no $\geq$ grade 2 urinary urgency during RT ( $11.1 \%$ (placebo) vs $0 \%$ (metformin), $\mathrm{p}=0.052)$. Those receiving metformin had no increased urinary frequency during RT $(16.7 \%$ (placebo) vs. $0 \%$ (metformin), $p=0.033$ ). There were no differences in overall $\geq$ grade $2 \mathrm{GU}$ toxicity between arms (19.0\% (placebo) vs. $9.4 \%$ (metformin), $p=0.30$ ). There were no $\geq$ grade 3 overall $\mathrm{Gl}, \mathrm{GU}$ or ADT-associated toxicities reported during follow-up.

\section{Discussion}

Metformin has been postulated to stabilize or prevent some of the adverse metabolic effects of ADT. However, there is paucity of data regarding the toxicity profile of the combination of metformin concurrent to pelvic/prostate radiotherapy. In this study, we did not detect any evidence that metformin increased acute or subacute gastrointestinal or genitourinary toxicity.

Furthermore, toxicity rates reported herein align with previously reported acute GI and GU toxicities of prostate radiotherapy using modern VMAT or IMRT techniques. ${ }^{16-18}$ Although data is limited due to the relatively recent introduction of VMAT or IMRT, $\geq$ grade 2 acute $\mathrm{GI}$ toxicities are reported at rates of 2.3$4 \%$ and $\geq$ grade 2 acute GU toxicities are reported at rates of $7-8.5 \% .{ }^{16,17}$ Overall combined $\geq$ grade 2 acute $\mathrm{GI}$ and $\mathrm{GU}$ toxicities combined rates reported were $9.7 \% .{ }^{16}$

These interim results detected no increases in GI or GU toxicity with metformin added to ADT and pelvic radiotherapy. The titration period of metformin, which coincided with the neoadjuvant phase of ADT, likely 
provided adequate time for participants to adjust to GI side effects of metformin prior to the start of radiotherapy. Further follow-up is merited.

\section{Conclusions}

Metformin added to radiotherapy and ADT did not increase rates of $\geq$ grade $2 \mathrm{GI}$ or GU toxicity. While these findings are preliminary, the addition of metformin to RT and ADT appears to be safe and welltolerated.

\section{List Of Abbreviations}

Androgen deprivation therapy (ADT), prostate cancer (PCa), gastrointestinal (GI), randomized controlled trial (RCT), Eastern Cooperative Oncology Group (ECOG), Hemoglobin A1c (HgbA1c), gleason score (GS), external beam radiotherapy (EBRT), luteinizing hormone-releasing hormone (LHRH), intensity modulated radiotherapy (IMRT), volumetric modulated arc therapy (VMAT), Common Terminology Criteria for Adverse Events (CTCAE)

\section{Declarations}

\section{Ethics Approval and Consent to Participate:}

This study was conducted with the prior written authorization of the Research Ethics Boards of the University of Manitoba and the University of Alberta. All patients provided written informed consent prior to undergoing study related procedures and all study conduct was in accordance with the Tri-Council Policy Statement Guidelines for Research Involving Human Subjects. This study was registered on a clinical trials registry: ClinicalTrials.gov Trial identifier NCT01996696.

\section{Availability of Supporting Data:}

The datasets generated and/or analysed during the current study are not publicly available due to it being an interim analysis with ongoing data collection for the study, but will be available in the future following final analysis from the corresponding author on reasonable request

\section{Competing Interests:}

HQ discloses consultant fees from Sanofi, Bayer, Astellas, Pfizer, Merck, and Janssen outside the submitted work. BD discloses personal fees from Janssen, Bayer, Astellas, Ferring outside the submitted work.

\section{Funding:}

Funding for this study was provided by competitive grants awarded by the CancerCare Manitoba Foundation and the Alberta Cancer Foundation. 


\section{Authors' Contributions:}

$\mathrm{JK}$ and NU conceptualized and designed the study protocol, conducted data analysis, drafting and final approval of the edited manuscript. JK and NU obtained grant funding for the study. MM carried out data analysis and drafting/editing of the manuscript. NU, MM, AO, RK, AD, WH, SA, HQ, DY, MP, GS, DP, DR, SG, and JK edited the manuscript. SG contributed to statistical analysis and manuscript review/editing. All authors reviewed and approved the manuscript prior to submission.

\section{Acknowledgements:}

Not Applicable

\section{References}

1. Rick FG, Schally A V. Bench-to-bedside development of agonists and antagonists of luteinizing hormone-releasing hormone for treatment of advanced prostate cancer. Urol Oncol Semin Orig Investig. 2015;33(6):270-274. doi:10.1016/j.urolonc.2014.11.006

2. Bolla $M$, Collette $L$, Blank $L$, et al. Long-term results with immediate androgen suppression and external irradiation in patients with locally advanced prostate cancer (an EORTC study): A phase III randomised trial. Lancet. 2002;360(9327):103-108. doi:10.1016/S0140-6736(02)09408-4

3. Bolla M, De Reijke TM, Van Tienhoven G, et al. Duration of androgen suppression in the treatment of prostate cancer. N Engl J Med. 2009;360(24):2516-2527. doi:10.1056/NEJMoa0810095

4. D'Amico A V, Manola J, Loffredo M, Renshaw AA, DellaCroce A, Kantoff PW. 6-Month Androgen Suppression Plus Radiation Therapy vs Radiation Therapy Alone for Patients With Clinically Localized Prostate Cancer: A Randomized Controlled Trial. JAMA. 2004;(7):821-827.

5. Roach M, Bae K, Speight J, et al. Short-Term Neoadjuvant Androgen Deprivation Therapy and External-Beam Radiotherapy for Locally Advanced Prostate Cancer: Long-Term Results of RTOG 8610. J Clin Oncol. 2008;(4):585-591.

6. Dockery F, Bulpitt CJ, Agarwal S, Donaldson M, Rajkumar C. Testosterone suppression in men with prostate cancer leads to an increase in arterial stiffness and hyperinsulinaemia. Clin Sci. 2003;104(2):195-201. doi:10.1042/CS20020209

7. Basaria S, Muller DC, Carducci MA, Egan J, Dobs AS. Hyperglycemia and insulin resistance in men with prostate carcinoma who receive androgen-deprivation therapy. Cancer. 2006;106(3):581-588. doi:10.1002/cncr.21642

8. Basaria S. Androgen deprivation therapy, insulin resistance, and cardiovascular mortality: An inconvenient truth. J Androl. 2008;29(5):534-539. doi:10.2164/jandrol.108.005454

9. Braga-Basaria M, Dobs AS, Muller DC, et al. Metabolic syndrome in men with prostate cancer undergoing long-term androgen-deprivation therapy. J Clin Oncol. 2006;24(24):3979-3983. doi:10.1200/JCO.2006.05.9741 
10. Smith MR, Finkelstein JS, McGovern FJ, et al. Changes in body composition during androgen deprivation therapy for prostate cancer. J Clin Endocrinol Metab. 2002;87(2):599-603. doi:10.1210/jcem.87.2.8299

11. Alberti KGMM, Eckel RH, Grundy SM, et al. Harmonizing the metabolic syndrome: A joint interim statement of the international diabetes federation task force on epidemiology and prevention; National heart, lung, and blood institute; American heart association; World heart federation; International atherosclerosis society; And international association for the study of obesity. Circulation. 2009;120(16):1640-1645. doi:10.1161/CIRCULATIONAHA.109.192644

12. Bailey CJ. Biguanides and NIDDM. Diabetes Care. 1992;15(6):755-772. doi:10.2337/diacare.15.6.755

13. Bailey CJ, Turner RC. Metformin. N Engl J Med. 1996;334(9):574-579. doi:10.1056/nejm199602293340906

14. Penney KL, Stampfer MJ. Time is ripe for a randomized trial of metformin in clinically localized prostate cancer. J Clin Oncol. 2013;31(25):3054-3055. doi:10.1200/JC0.2013.50.7715

15. Gillessen S, Gilson C, James N, Adler A, Sydes MR, Clarke N. Repurposing Metformin as Therapy for Prostate Cancer within the STAMPEDE Trial Platform. Eur Urol. 2016;70(6):906-908. doi:10.1016/j.eururo.2016.07.015

16. Michalski JM, Yan Y, Watkins-Bruner D, et al. Preliminary Toxicity Analysis of 3-Dimensional Conformal Radiation Therapy Versus Intensity Modulated Radiation Therapy on the High-Dose Arm of the Radiation Therapy Oncology Group 0126 Prostate Cancer Trial. Int J Radiat Oncol Biol Phys. 2013;(5):932-938.

17. Dearnaley D, Syndikus I, Sumo G, et al. Conventional versus hypofractionated high-dose intensitymodulated radiotherapy for prostate cancer: Preliminary safety results from the $\mathrm{CHHiP}$ randomised controlled trial. Lancet Oncol. 2012;13(1):43-54. doi:10.1016/S1470-2045(11)70293-5

18. Holch P, Henry AM, Davidson S, et al. Acute and Late Adverse Events Associated With Radical Radiation Therapy Prostate Cancer Treatment: A Systematic Review of Clinician and Patient Toxicity Reporting in Randomized Controlled Trials. Int J Radiat Oncol Biol Phys. 2017;97(3):495-510. doi:10.1016/j.jijrobp.2016.11.008

\section{Table}


Table 1

Baseline Characteristics of the Patients.

\begin{tabular}{|c|c|c|c|}
\hline Characteristic* & Metformin $(n=39)$ & Placebo $(n=42)$ & p-value \\
\hline Age & $71(56-82)$ & $73(49-86)$ & 0.22 \\
\hline Weight (kg) & $95.5(62.7-157.0)$ & $91.6(71.8-126.1)$ & 0.24 \\
\hline Waist Circumference (cm) & $110(81.5-185)$ & $109(92-185)$ & 0.60 \\
\hline BMI $\left(\mathrm{kg} / \mathrm{m}^{2}\right)$ & $30.3(22.2-52.5)$ & $29.8(23.7-40.4)$ & 0.40 \\
\hline Mean SBP $(\mathrm{mmHg})$ & $145(108-179)$ & $142.5(103-173.5)$ & 0.92 \\
\hline $\mathrm{HbA1C}(\%)$ & $5.6(5.1-6.4)$ & $5.6(4.9-6.4)$ & 0.95 \\
\hline Smoking Pack-Year-History & $15(0-107.5)$ & $20(0-75)$ & 0.56 \\
\hline Marital Status - no. (\%) married & $31(79.5)$ & $37(88.1)$ & 0.29 \\
\hline ECOG & $0(0-1)$ & $0(0-1)$ & 0.59 \\
\hline Total IPSS & $11(0-31)$ & $10(0-22)$ & 0.86 \\
\hline Gleason Score & $9(7-9)$ & $8(7-9)$ & 0.44 \\
\hline \% Biopsy Cores Positive & $7(2-14)$ & $7(2-12)$ & 0.62 \\
\hline \multicolumn{4}{|l|}{ Clinical T-Stage - no. (\%) } \\
\hline T1 & $13(33)$ & $13(31)$ & 0.87 \\
\hline $\mathrm{T} 2$ & $16(41)$ & $16(38)$ & \\
\hline T3 & $10(26)$ & $13(31)$ & \\
\hline Pelvic Nodal Irradiation - no. (\%) & $30(71)$ & $33(79)$ & 0.82 \\
\hline \multicolumn{4}{|l|}{ Prostate Boost Type - no. (\%) } \\
\hline Standard Fractionation $(76 / 38$ to $78 / 39)$ & $24(62)$ & $24(57)$ & 0.90 \\
\hline Hypofractionated $(60 / 20,70 / 28,72.8 / 28)$ & $11(28)$ & $13(31)$ & \\
\hline Interstitial Brachytherapy Boost & $4(10)$ & $5(12)$ & \\
\hline
\end{tabular}

*Values given as mean (range) unless otherwise indicated.

Abbreviations: SBP = Systolic Blood Pressure; HbA1c = Hemoglobin A1C; ECOG = Eastern Cooperative Oncology Group; IPSS = International Prostate Symptom Score

\section{Figures}



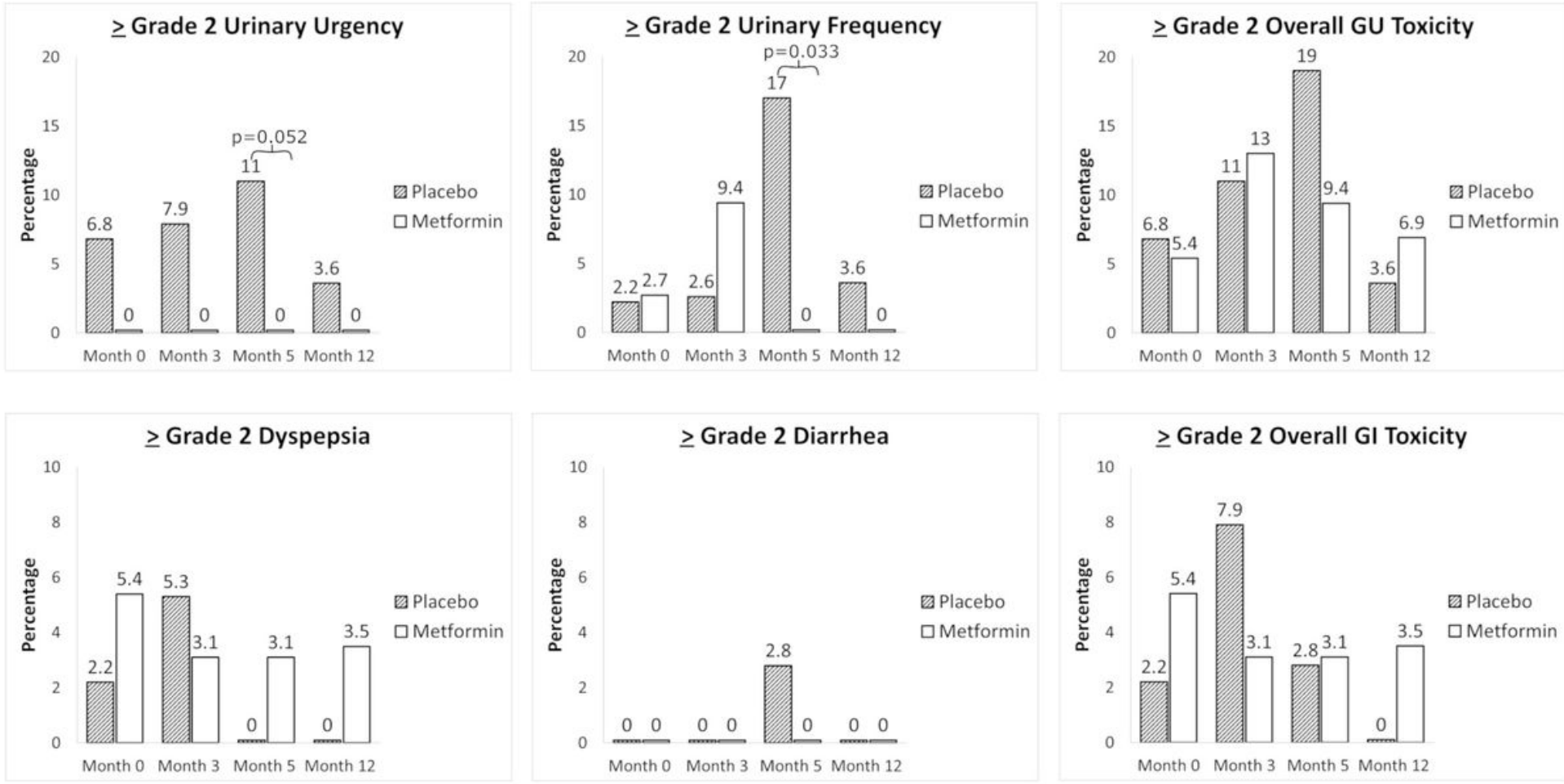

Figure 1

Genitourinary and gastrointestinal toxicity by treatment. 


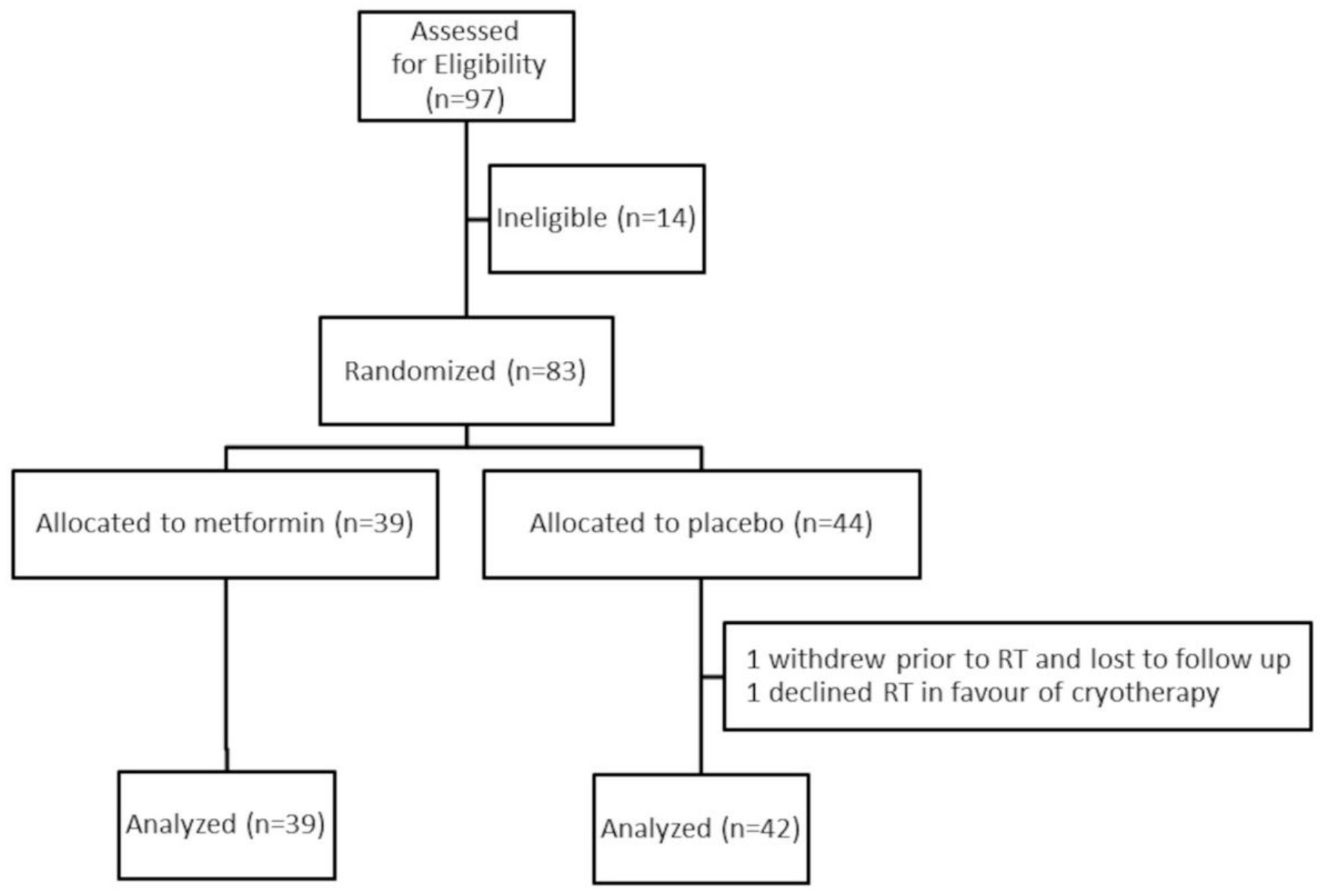

Figure 2

Enrollment and treatment allocation.

\section{Supplementary Files}

This is a list of supplementary files associated with this preprint. Click to download.

- SupplementalTable111Feb2021JK.xls 